\title{
Relative humidity multi-point optical sensors system based on Fast Fourier multiplexing technique
}

\author{
A. Lopez-Aldaba*a, D. Lopez-Torres ${ }^{\mathrm{a}}$, C. Elosua ${ }^{\mathrm{a}}$, J.-L. Auguste ${ }^{\mathrm{b}}$, R. Jamier ${ }^{\mathrm{b}}$, P. Roy ${ }^{\mathrm{b}}$, F.J. Arregui ${ }^{\mathrm{a}}$ \\ M. Lopez-Amo ${ }^{\mathrm{a}}$ \\ aniversidad Pública de Navarra, Dept. of Electrical and Electronic Engineering and Institute Of \\ Smart Cities (ISC), Campus Arrosadia S/N, E-31006, Pamplona, Spain; \\ ${ }^{b}$ Xlim, Fibre Photonics Department, UMR CNRS/University of Limoges 7252, 123 Avenue \\ Albert Thomas, 87060 Limoges Cedex, France \\ * aitor.lopez@unavara.es; phone +3494816 9841; fax +34948169720
}

\begin{abstract}
In this paper, a new multipoint optical fiber system for relative humidity measurements based on $\mathrm{SnO}_{2}$ - $\mathrm{FP}$ (Fabry-Pérot) sensing heads and an optical interrogator as single active device is presented and characterized. The interrogation of the sensing heads is carried out by monitoring the Fast Fourier Transform phase variations of the FP (Fabry-Pérot) interference frequencies. This method allows to multiplex several sensors with different wavelength spacing interference pattern. The sensors operate within a wide humidity range (20\%-90\% relative humidity) with low crosstalk between them. Five sensing heads have been measured using two different channels of the optical interrogator. The availability of four channels in the interrogator allows to multiplex a higher number of sensors, reducing proportionally the cost of each sensing point.
\end{abstract}

Keywords: Photonic crystal fiber, microstructured optical fiber, sputtering, fiber sensor, humidity sensing, Fast Fourier Transform, multiplexing.

\section{INTRODUCTION}

Microstructured optical fibers (MOFs) have shown improved characteristics over conventional optical fibers and a great potential for sensing applications [1]. For instance, several pure silica suspended-core fibers have been used in temperature and curvature sensing [2] or relative humidity (RH) sensing [3].

Fiber based optical Fabry-Pérot (FP) interferometers are a quite popular sensor configuration due to their compactness, simple configuration, flexibility in tuning sensitivity and dynamic range. FP cavities composed by MOFs are also common structures: a hybrid structure that used a MOF as the guiding fiber and cascade it with a hollow-core fiber and a single mode fiber (SMF), for high-temperature sensing, was demonstrated [4].

Among optical fiber sensors, nanocoated based sensors have recently experienced a remarkable development [5]. Furthermore, new techniques in chemical deposition, such as sputtering [6], enable to control the morphology and thickness of the deposited coatings with high accuracy, and as a consequence, the final properties (sensitivity, kinetics) of the sensor.

Multiplexing interferometric sensors is a major target in the sensing field, allowing to perform multi-point and multiparameter measurements within the scheme, reducing significantly the economic cost of the system. Multiplexing some Fabry-Pérot interferometric sensors present several difficulties due to their low reflectivity (4\%) and their cavity length limitation. Several approaches have been demonstrated during the last years: spatial-frequency-division multiplexing (SFDM) and coarse-wavelength-division multiplexing (CWDM) schemes [7] or in-line FP cavities based on FBGs reflectometers [8].

In this paper, a five- $\mathrm{SnO}_{2}$-FP-interferometric-multiplexing system for relative humidity is presented and characterized. Using a commercial FBG interrogator with a MATLAB based software, five different RH sensors are simultaneously and independently measured. By monitoring the Fast Fourier Transform (FFT) phase variations of the FP interference frequencies, an experimental study of the sensors response and their crosstalk is presented. This measuring method is independent of the signal amplitude and avoids the necessity of tracking the wavelength evolution in the spectrum, which becomes a problem when several interferometric contributions come up.

25th International Conference on Optical Fiber Sensors, edited by Youngjoo Chung, Wei Jin,

Byoungho Lee, John Canning, Kentaro Nakamura, Libo Yuan, Proc. of SPIE Vol. 10323,

103233L · C 2017 SPIE · CCC code: 0277-786X/17/\$18 · doi: 10.1117/12.2265174 


\section{EXPERIMENTAL SET-UP AND OPERATION PRINCIPLE}

The Fabry-Pérot interferometers were fabricated by splicing a single mode fiber to a novel four-bridge MOF, with its end cleaved, following the method shown in [3]. The MOF-FP's optical fiber core was used as the substrate in a $\mathrm{SnO}_{2}$ coatingDC-Sputter deposition process also demonstrated in [3]. $\mathrm{SnO}_{2}$ coating is highly sensitive to changes in the refractive index of the surrounding environment [9], which in this case are produced by relative humidity variations.

Due to the direct relation between the MOF's cavity length and the obtained sensor optical spectrum interference's periodicity $\left(\Delta \lambda=\frac{\lambda^{2}}{2 n d}\right.$; where $\Delta \lambda$ : optical spectrum wavelength spacing, $\lambda$ : working wavelength, $\mathrm{n}$ : refractive index and d: MOF cavity length) different sensing heads with different interference patterns can be achieved by choosing properly the MOF's lengths.

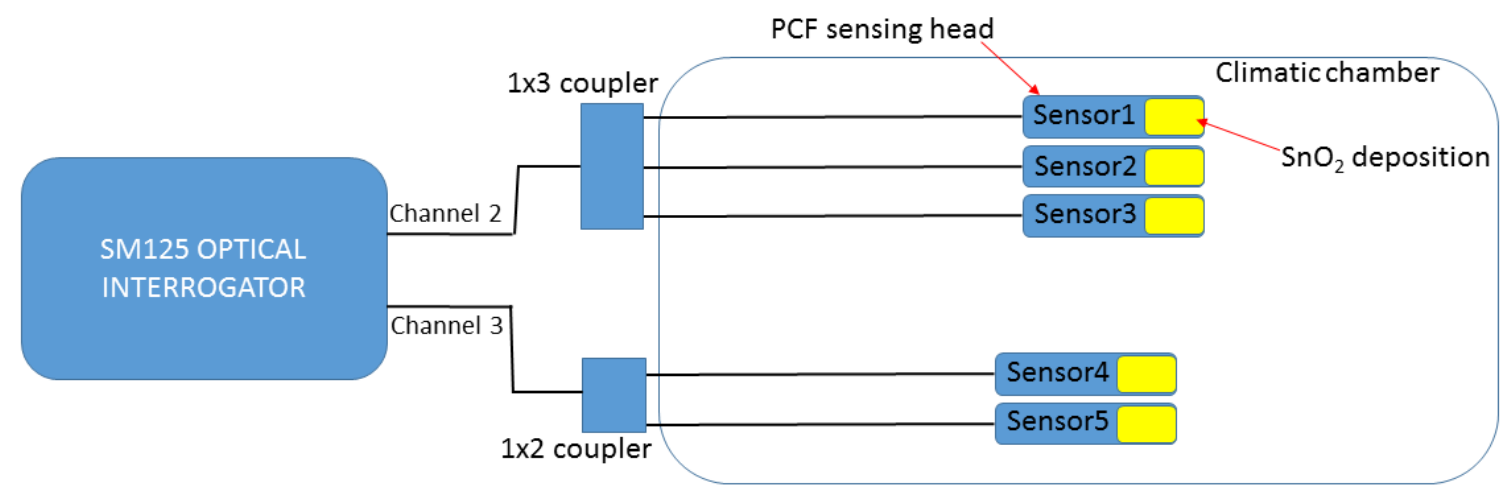

Figure 1. Experimental setup of the proposed system.

Figure 1 presents the experimental set-up used to characterize the four-bridge MOF Fabry-Pérot based sensing heads and the $\mathrm{SnO}_{2}$-FP final sensors. A commercial interrogator equipment for FBGs based sensors (Smartec SM125) was used to illuminate the network and also to analyze the spectra of the signals reflected in the sensors. The interrogating sensor equipment has a sampling frequency of $1 \mathrm{~Hz}$ [10], four different channels and it is remotely controlled through a MATLAB software that also executes a FFT real time analysis. The sensing heads were placed inside a climatic chamber where humidity ranges from $20 \%$ to $90 \%$ were applied at a constant temperature of $25^{\circ} \mathrm{C}$ to evaluate their response to this magnitude.

Sensor1, Sensor2 and Sensor 3 were multiplexed through a 1x3 coupler into the optical interrogator's Channel 2 while Sensor4 and Sensor5 were multiplexed into Chanel 3 through a 1x2 coupler.

\section{EXPERIMENTAL RESULTS}

Five different sensors were fabricated by fusing different lengths of MOF fiber to a single mode fiber. Lengths $\mathrm{d} 1=266 \mu \mathrm{m}$, $\mathrm{d} 2=800 \mu \mathrm{m}, \mathrm{d} 3=540 \mu \mathrm{m}, \mathrm{d} 4=520 \mu \mathrm{m}$ and d5 $=460 \mu \mathrm{m}$ were used to create Sensor1, Sensor2, Sensor3, Sensor4 and Sensor5 respectively. As a consequence of these lengths, each sensor has its own interference fringe pattern: $\Delta \lambda 1=3 \mathrm{~nm}, \Delta \lambda 2=1 \mathrm{~nm}$, $\Delta \lambda 3=1.48 \mathrm{~nm}, \Delta \lambda 4=1.54 \mathrm{~nm}$ and $\Delta \lambda 5=1.74 \mathrm{~nm}$. The resultant optical signal measured in channel 2 and 3 can be seen in Figure 2 a).

Through the FFT analysis, each single optical interference is directly related to a one single FFT spatial frequency component. The FFT spatial frequencies for the studied sensors are located at Freq $1=0.55 \mathrm{~nm}^{-1}$, Freq $2=1 \mathrm{~nm}^{-1}$, Freq3 $=0.675 \mathrm{~nm}^{-1}$, Freq $4=0.65 \mathrm{~nm}^{-1}$ and Freq $5=0.575 \mathrm{~nm}^{-1}$, as can be seen in Figure $2 \mathrm{~b}$ ). It should be noticed the importance of the MOF length choice: if two sensors have similar optical interference pattern, their FFT spectrum would overlap leading to crosstalk between measurements (if they are close but not at the same spatial frequency) or making impossible to discriminate between them (if they are at the same spatial frequency).

Furthermore, as can be seen in Figure 2 b), due to the beat generated in the coupler, intermodulation products of the sensor's different spatial frequencies appear (i.e. located at $\sim 0.4 \mathrm{~nm}^{-1}$ in Channel 2). Since they are the results of various sensors' contributions, the crosstalk between them does not allow to measure independently. 

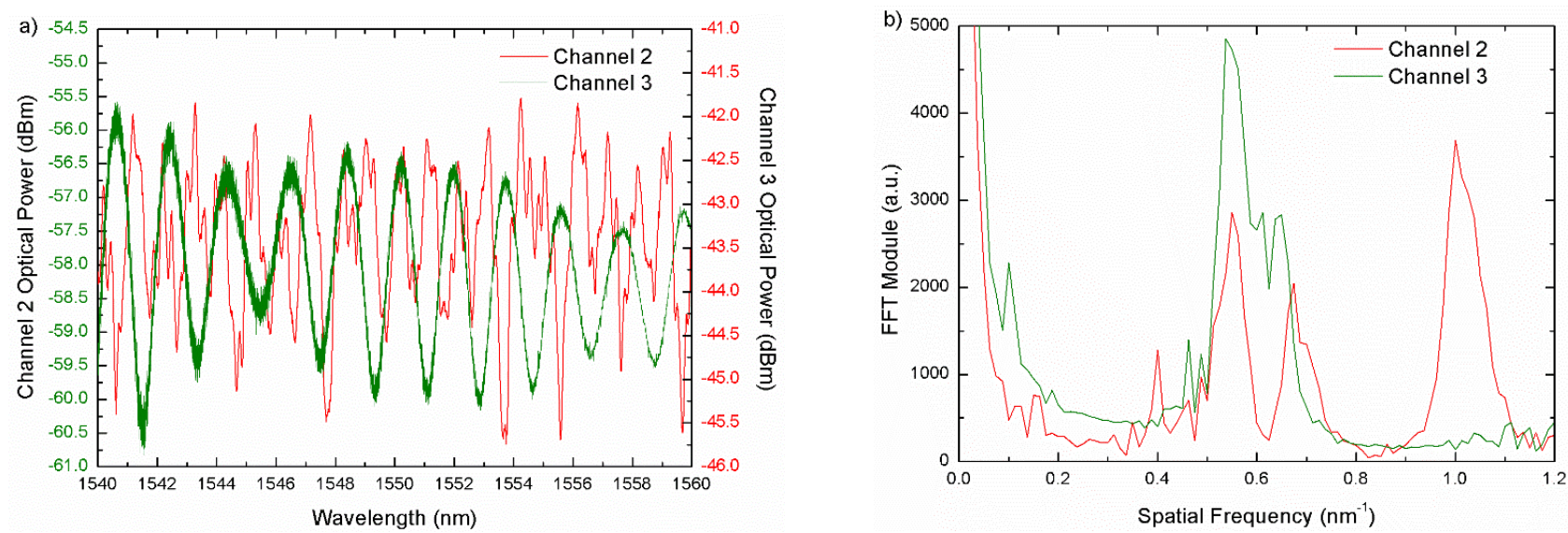

Figure 2: a) Measured optical spectrum of the sensors at $25^{\circ} \mathrm{C}$ and $40 \%$ humidity (room conditions) and b) their Fast Fourier Transform.

In order to verify the performance of the multi-SnO 2 -FP sensing system, the humidity-sensing performance of each sensor, with different RHs (constant $25^{\circ} \mathrm{C}$ ), was experimentally carried out. Figure 3 a) shows the experimental performance of Sensor1, Sensor 2 and Sensor 3 in Channel 2 with sensitivities of $0.012,0.013$ and $0.014 \pi \mathrm{rad} / \%$ RH respectively. Figure 3 b) shows the results for Sensor4 and Sensor5 in Channel 3 with sensitivities of 0.007 and $0.011 \pi \mathrm{rad} / \% \mathrm{RH}$ respectively.
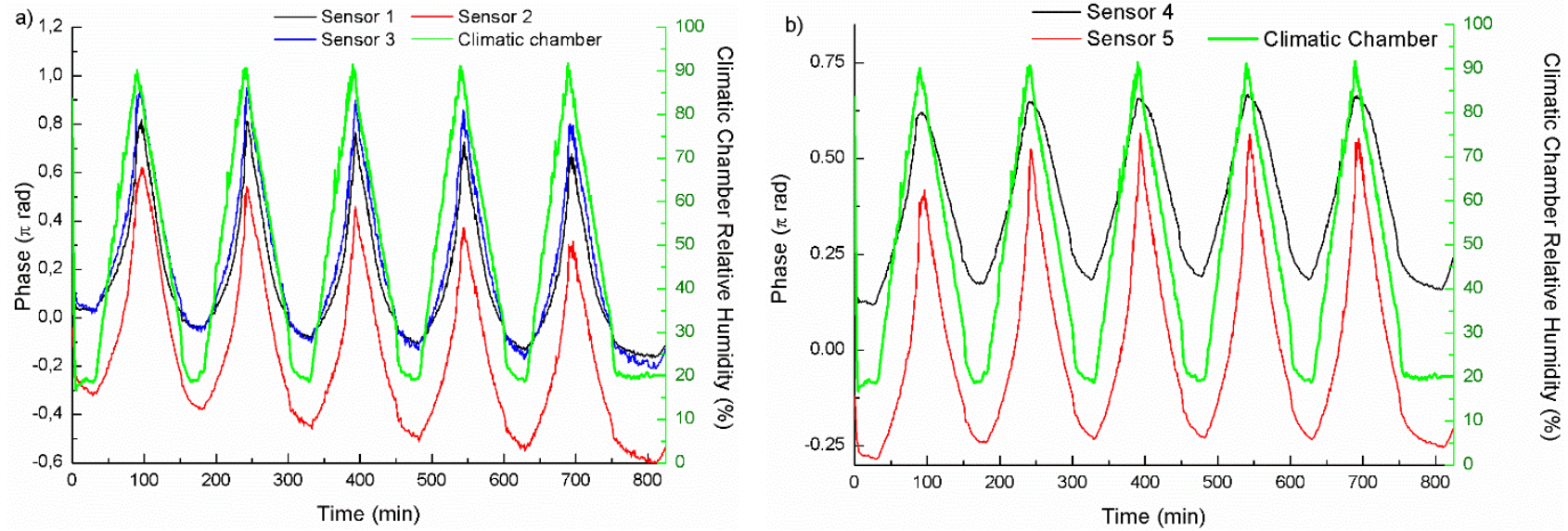

Figure 3. Sensor response to humidity: a) Channel 2, and b) Channel 3.
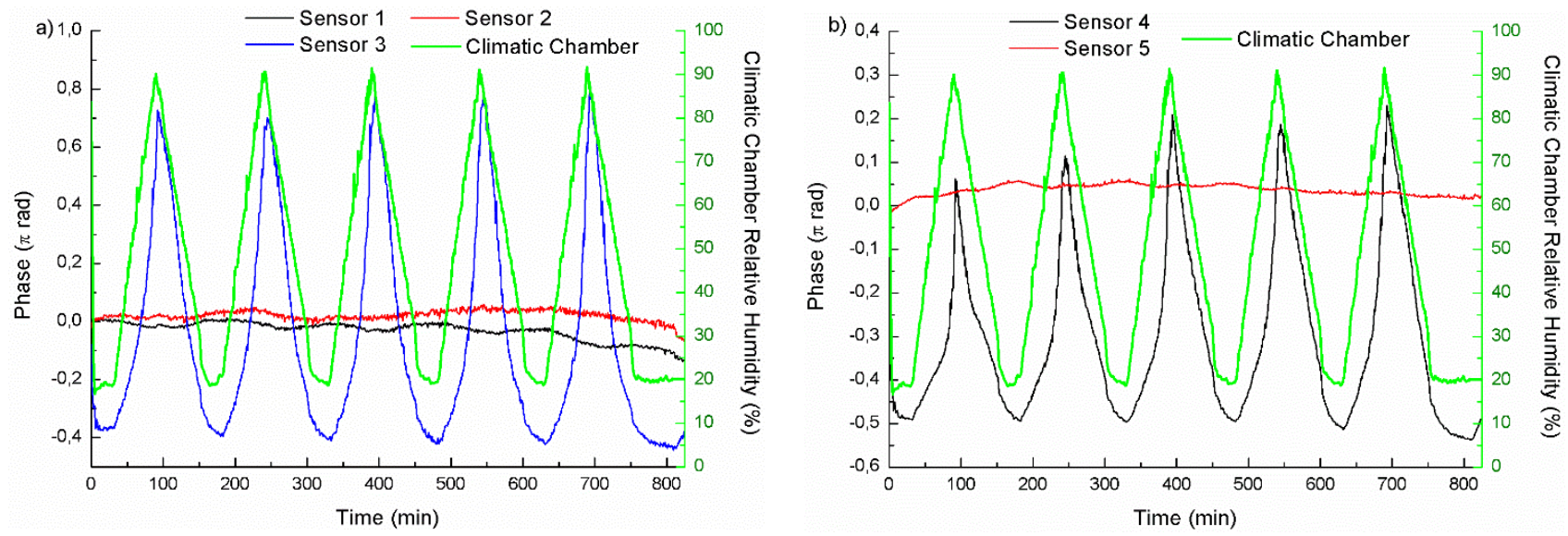

Figure 4. Sensors crosstalk: a) Channel 2, and b) Channel 3. 
A study of the crosstalk between sensors in the same channel was also carried out. One sensor per channel (Sensor3 for Channel 2 and Sensor4 for Channel 3) was placed inside the climatic chamber while the others stayed outside. Figure 4 shows the measured results. It has to be noticed that temperature variations outside the climatic chamber affected the measured phase for sensors 1,2 and 5 and they have been compensated.

\section{CONCLUSIONS}

To summarize, a new multi-point system for relative humidity measuring based on $\mathrm{SnO}_{2}$-FP (Fabry-Pérot) sensing heads and an optical interrogator have been proposed and experimentally demonstrated. The sensing head's interrogation has been carried out by monitoring the FFT phase variations of each sensor's spatial frequencies. This method is low-dependent of the signal amplitude and also avoids the need to track the optical spectrum evolution of maxima and minima, which is a handicap when multiple contributions are present. Five $\mathrm{SnO}_{2}$-FP sensing heads for relative humidity measurements have been multiplexed among two different channels of the optical interrogator. Due to the optical interrogator's features, having four different channels and a studied choice of the MOF's lengths, the number of sensing heads multiplexed with one single active device can be remarkably increased, decreasing significantly the economic cost of the system. Moreover, different parameters such as strain, temperature and RH can be simultaneously measured.

\section{ACKNOWLEDGMENTS}

The authors thank the Spanish Government projects TEC 2013-47264-C2-2-R, TEC2016-76021-C2-1-R, TEC201343679-R Innocampus and the INTERREG SUDOE Project SOE3/P2/P714 as well as to the FEDER Funds.

\section{REFERENCES}

[1] Pinto, A. M. R. and Lopez-Amo, M., "Photonic Crystal Fibers for Sensing Applications," Journal of Sensors 2012, 21 (2012).

[2] Frazao, O., Silva, S.F.O., Viegas, J., Baptista, J.M., Santos, J.L., Kobelke, J. and Schuster, K., "All Fiber MachZehnder Interferometer Based on Suspended Twin-Core Fiber," IEEE Photonic Tech L 22, 1300-1302 (2010).

[3] A. Lopez-Aldaba, D. Lopez-Torres, J. Ascorbe, S. Rota-Rodrigo, C. Elosua, M. Lopez-Amo, F. J. Arregui, J. M. Corres, J.-L. Auguste, R. Jamier and P. Roy; "SnO2-MOF-Fabry-Perot humidity optical sensor system based on fast Fourier transform technique". Proc. SPIE 9916, Sixth European Workshop on Optical Fibre Sensors, 99161T (2016).

[4] Choi, H., Park, K., Park, S., Paek, U., Lee, B. and Choi, E., "Miniature fiber-optic high temperature sensor based on a hybrid structured Fabry-Perot interferometer," Optics Letters, 2455-2457 (2008).

[5] Arregui, F.J., "Sensors Based on Nanostructured Materials". New York (NY): Springer, 2009.

[6] Stowell, M., Müller, J., Ruske, M., Lutz, M. and Linz, T., "RFsuperimposed DC and pulsed DC sputtering for deposition of transparent conductive oxides," Thin Solid Films, 7654-7657, (2007).

[7] Rao, Yun-Jiang. "Recent progress in fiber-optic extrinsic Fabry-Perot interferometric sensors." Optical Fiber Technology 12.3, 227-237. (2006).

[8] Wang, Z., Shen, F., Song, L., Wang, X., and Wang, A., "Multiplexed fiber Fabry-Perot interferometer sensors based on ultrashort Bragg gratings." IEEE Photonics Technology Letters 19.8, 622-624 (2007).

[9] Sanchez, P. , Zamarreño, C.R., Hernaez, M., Matias, I.R., Arregui, F.J., "Optical fiber refractometers based on Lossy Mode Resonances by means of SnO2 sputtered coatings," Sensors and Actuators, B: Chemical 202, 154159, (2014).

[10] Leandro, D., Bravo, M., Ortigosa A., Lopez-Amo M., "Real-time FFT analysis for interferometric sensors multiplexing," J Lightwave Technol. 33(2), 354-360, (2015). 